\title{
Factores sociodemográficos asociados a la mortalidad por suicidios en México, 2012-2016
}

\author{
Sociodemographic factors associated with suicide mortality rates in Mexico, 2012-2016
}

Claudio A. Dávila-Cervantes ${ }^{1 *}$ orcid.org/0000-0002-7656-3606

1 Facultad Latinoamericana de Ciencias Sociales (FLACSO). Ciudad de México, México

\section{Resumen}

Introducción: La mortalidad por suicidio se ha incrementado sostenida e ininterrumpidamente en México desde hace más de 40 años. Objetivo: El principal objetivo fue analizar los factores sociodemográficos asociados con la mortalidad por suicidio en México entre 2012 y 2016. Materiales y métodos: Se realizó un estudio de tipo observacional y transversal. Las bases de datos de defunciones se obtuvieron del Instituto Nacional de Estadística y Geografía en México para 2012-2016. Se ajustó un modelo de regresión logística binomial para analizar qué factores sociodemográficos se relacionan con la posibilidad de que las personas fallezcan por suicidio en México. Resultados: Las mujeres tuvieron una menor posibilidad de fallecer por suicidio y conforme aumenta la edad, las personas tienen menos posibilidad de suicidarse. Tener algún grado de escolaridad o realizar una actividad económica incrementó la posibilidad de fallecer por suicidio. Conclusiones: Se espera que este estudio aporte mayor evidencia que ayude a generar políticas de prevención, atención oportuna, y la disminución del impacto de esta causa de muerte en la sociedad.

Palabras clave: Suicidio; México; mortalidad; factores de riesgo. (Fuente: DeCS, Bireme).

\begin{abstract}
Introduction: Mortality due to suicide has increased steadily and continuously in Mexico for more than 40 years. Objective: The main objective was to analyze the sociodemographic factors associated with suicide mortality rates in Mexico between 2012 and 2016. Materials and methods: An observational and cross-sectional study was conducted. Death databases were obtained from the National Institute of Statistics and Geography in Mexico for 2012-2016. A binomial logistic regression model was adjusted to analyze which sociodemographic factors are related to the possibility that people die of suicide in Mexico. Results: Women had a lower chance of dying from suicide, and as age increases, people are less likely to commit suicide. Having some level of schooling or performing an economic activity increased the possibility of death by suicide. Conclusions: This study is expected to provide more evidence to help generate prevention policies, timely care, and the reduction of the impact of suicide on society.
\end{abstract}

Key words: Suicide; Mexico; cause of death; risk factors. (Source: DeCS, Bireme).

\footnotetext{
*Autor de correspondencia Claudio A. Dávila Cervantes e-mail: claudio.davila@flacso.edu.mx
} 


\section{Introducción}

El suicidio es uno de los principales problemas de salud pública, en 2012 fue la decimoquinta causa de muerte a nivel mundial; representó, casi la mitad de todas las muertes violentas para hombres y más de $70 \%$ para mujeres(1). Esto se tradujo en casi un millón de víctimas, $\mathrm{y}$ altos costos sociales $\mathrm{y}$ económicos(2). Tres de cada cuatro suicidios ocurren en países de ingresos medios y bajos, donde la identificación temprana se complica debido a que los recursos y servicios son escasos, limitados, los tratamientos y apoyos son insuficientes ${ }^{(1)}$. Latinoamérica $y$ el Caribe presentan tasas relativamente bajas de suicidio $(6,1$ defunciones por 100.000 habitantes)(1), pero en los últimos 20 años se han incrementado en toda la región(2).

Entre 1970 y 2016 la tasa de mortalidad por suicidios en jóvenes, casi se quintuplicó, pasando de 1,13 en 1970; 2,63 en 1990, a 5,2 en 2016, donde se presentaron 4,4 suicidios masculinos por cada femenino; para la población de quince a veintinueve años es la tercera causa de muerte en ambos sexos, después de los homicidios y accidentes de vehículo automotor; constituye una de las principales causas de años de vida perdidos por muerte prematura(3-5).

Los factores de riesgo de suicidio más recurrentes en el país son el consumo nocivo de alcohol o drogas, dolores y enfermedades crónicas, desórdenes psiquiátricos, eventos negativos en la vida, entre

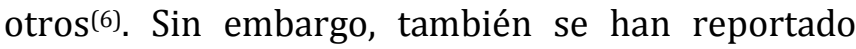
factores sociodemográficos que se asocian con un mayor riesgo de suicidio como el sexo, la edad, la escolaridad, el estatus de unión y la situación laboral. Dada la problemática y los escasos estudios en México, el objetivo de esta investigación es analizar los factores sociodemográficos asociados con la mortalidad por suicidio en México a nivel nacional entre 2012 y 2016.

\section{Materiales y métodos}

Se realizó un estudio de tipo observacional y transversal. Las bases de datos de defunciones se obtuvieron del Instituto Nacional de Estadística y Geografía (INEGI) en México para 2012-2016. Se adoptó la definición de suicidios de la Clasificación Internacional de Enfermedades (CIE-10) (códigos X60-X84; Y870)(7).
Se estudió los fallecimientos oficiales registrados entre 2012-2016. Se realizó un análisis descriptivo bivariado para identificar los fallecimientos por suicidios y los factores sociodemográficos asociados, usando tablas de contingencia y la prueba de independencia Ji-Cuadrado. La variable bajo estudio fue si la causa de muerte fue suicidio o no. Las características sociodemográficas analizadas fueron: sexo, edad, escolaridad, situación de unión y condición de actividad. Se ajustó un modelo de regresión logística binomial (MRLB) para analizar qué factores sociodemográficos se asocian con la posibilidad de que las personas hayan fallecido por suicidio en México. El año fue la variable de control. Se incluyeron dos interacciones entre edad y sexo, y entre sexo y condición de actividad. Se calcularon los coeficientes estandarizados para conocer cuáles variables independientes tuvieron mayor y menor efecto sobre el fallecimiento por suicidio. Se excluyeron del análisis los suicidios con registros incompletos en alguna de las variables bajo estudio $(14,1 \%$ del total de suicidios registrados). Los casos excluidos presentaron un menor porcentaje de actividades económicas, se encontraron menos en unión y tuvieron una menor escolaridad. Se trabajó con un nivel de significancia de $5 \%$. Los cálculos se realizaron con el programa STATA V. 14.1.

\section{Consideraciones éticas}

Se realizó un estudio con información proveniente de los certificados de defunción registrados $\mathrm{y}$ publicados por INEGI bajo el proyecto estadístico MEX-INEGI.40.202.06-EVM. Con ello que se garantiza la confidencialidad de los sujetos bajo estudio y no se tienen conflictos éticos.

\section{Resultados}

Entre 2012 y 2016 se registraron 30.591 suicidios en México, de los cuales $81 \%$ fueron masculinos. Poco más de $80 \%$ de los suicidios ocurrió en menores de 50 años (Tabla 1); en mujeres, más de la mitad ocurrieron antes de los 30 años; mientras que 8,2\% se presentaron en hombres de 65 años o más. En cuanto a la escolaridad, la mayor incidencia de suicidio se dio en aquellas personas con primaria o secundaria. No se observó una diferencia en la incidencia de suicidio según el estado civil. Para la variable de actividad económica se puede ver un comportamiento opuesto entre hombres y mujeres (por ello se decidió realizar una interacción entre las variables de sexo y actividad económica). En cuanto 
al método elegido para llevar a cabo el suicidio, principalmente fue el ahorcamiento seguido por el envenenamiento en mujeres y el uso de arma de fuego en hombres. Se encontró que existe una relación estadísticamente significativa entre todas las variables en estudio y el suicidio.

Tabla 1. Características sociodemográficas de las defunciones por suicidio en México por sexo, 2012-2016

\begin{tabular}{|c|c|c|c|}
\hline Características & $\begin{array}{c}\text { Hombres } \\
\mathrm{n}=21.373(81,4 \%)\end{array}$ & $\begin{array}{c}\text { Mujeres } \\
\mathrm{n}=4.906(18,6 \%)\end{array}$ & $\begin{array}{c}\text { Total } \\
\mathrm{n}=26.279\end{array}$ \\
\hline \multicolumn{4}{|l|}{ Edad* } \\
\hline $15-29$ años & 41,3 & 52,0 & 43,3 \\
\hline $30-49$ años & 37,9 & 34,0 & 37,2 \\
\hline $50-64$ años & 12,6 & 10,7 & 12,2 \\
\hline $65+$ años & 8,2 & 3,3 & 7,3 \\
\hline \multicolumn{4}{|l|}{ Nivel de escolaridad* } \\
\hline Sin escolaridad & 19,8 & 13,5 & 18,6 \\
\hline Primaria & 29,5 & 23,5 & 28,4 \\
\hline Secundaria & 32,7 & 37,1 & 33,6 \\
\hline Bachillerato o más & 18,0 & 25,9 & 19,5 \\
\hline \multicolumn{4}{|l|}{ En unión* } \\
\hline No & 49,3 & 52,4 & 49,9 \\
\hline $\mathrm{Si}$ & 50,7 & 47,6 & 50,1 \\
\hline \multicolumn{4}{|l|}{ Realiza actividad económica* } \\
\hline No & 20,3 & 70,1 & 29,6 \\
\hline $\mathrm{Si}$ & 79,7 & 29,9 & 70,4 \\
\hline \multicolumn{4}{|l|}{ Método* } \\
\hline Envenenamiento & 6,1 & 20,4 & 8,8 \\
\hline Ahorcamiento & 80,7 & 70,9 & 78,8 \\
\hline Ahogamiento & 0,2 & 0,6 & 0,3 \\
\hline Arma de fuego & 10,2 & 3,9 & 9,0 \\
\hline Objetos punzocortante & 1,1 & 0,9 & 1,1 \\
\hline Quemaduras & 0,2 & 0,3 & 0,2 \\
\hline Arrojarse & 0,9 & 1,7 & 1,0 \\
\hline Otros medios & 0,7 & 1,3 & 0,8 \\
\hline
\end{tabular}

* Significancia de la prueba de independencia Ji-Cuadrada, $\mathbf{p}<0,001$

Fuente: Elaboración propia con base en estadísticas vitales de mortalidad de INEGI, 2012-2016

El MRLB mostró que las mujeres tienen una menor posibilidad de fallecer por suicidio (Tabla 2). La edad a la cual suceden los suicidios varía según el sexo de la persona, sin embargo, se observó una disminución de suicidios a medida que aumenta la edad, esta tendencia es mayor en mujeres. Tener algún grado de escolaridad incrementó la posibilidad de morir por suicidio. La tendencia general indica que si las personas realizan una actividad económica, incrementan la posibilidad de fallecer por suicidio, sin embargo, esta condición varía según el sexo, pues en las mujeres se da una relación inversa. En 2014 y 2015 se observó una mayor propensión a fallecer por suicidio en el país con respecto a 2012. Las variables que tuvieron un mayor efecto sobre el fallecimiento por suicidio (ver coeficientes estandarizados) fueron: edad de 65 y más, mujeres de 65 años y más, y la edad de 50 a 64 años (todas disminuyeron la posibilidad de fallecer por suicidio). Aquellas variables que tuvieron un menor efecto sobre la mortalidad por suicidio fueron: el año 2015, las mujeres económicamente activas y el año 2014. Finalmente, las variables que más afectaron el incremento de los momios de fallecer por suicidio fueron: tener una escolaridad primaria, una escolaridad secundaria y realizar una actividad económica. 
Tabla 2. Resultados del modelo regresión logística binomial del fallecimiento por suicidio en México, 2012-2016

\begin{tabular}{|c|c|c|c|c|c|}
\hline Características & $\beta$ & Odds Ratio & IC $95 \%$ & P - valor & $\begin{array}{c}\text { Coeficientes } \\
\text { estandarizados }\end{array}$ \\
\hline \multicolumn{6}{|l|}{ Sexo } \\
\hline Hombre+ & - & - & - & - & \\
\hline Mujer & $-0,152$ & 0,859 & {$[0,81,0,91]$} & $\mathrm{p}<0,0001$ & $-0,076$ \\
\hline \multicolumn{6}{|l|}{ Edad } \\
\hline 15 - 29 años+ & - & - & - & - & \\
\hline 30 - 49 años & $-0,869$ & 0,420 & {$[0,41,0,43]$} & $\mathrm{p}<0,0001$ & $-0,295$ \\
\hline 50 - 64 años & $-2,220$ & 0,109 & {$[0,10,0,11]$} & $\mathrm{p}<0,0001$ & $-0,898$ \\
\hline $65+$ años & $-3,455$ & 0,032 & {$[0,03,0,03]$} & $\mathrm{p}<0,0001$ & $-1,690$ \\
\hline \multicolumn{6}{|l|}{ Sexo*Edad } \\
\hline Hombre de 15 - 29 años+ & - & - & - & - & \\
\hline Mujer de 30 - 49 años & $-0,665$ & 0,514 & {$[0,48,0,55]$} & $\mathrm{p}<0,0001$ & $-0,134$ \\
\hline Mujer de 50 - 64 años & $-1,089$ & 0,337 & {$[0,30,0,37]$} & $\mathrm{p}<0,0001$ & $-0,306$ \\
\hline Mujer de 65 años o más & $-2,187$ & 0,112 & {$[0,09,0,13]$} & $\mathrm{p}<0,0001$ & $-1,007$ \\
\hline \multicolumn{6}{|l|}{ Nivel de escolaridad } \\
\hline Sin escolaridad+ & - & - & - & - & \\
\hline Primaria & 0,330 & 1,391 & {$[1,34,1,44]$} & $\mathrm{p}<0,0001$ & 0,139 \\
\hline Secundaria & 0,402 & 1,495 & {$[1,44,1,55]$} & $\mathrm{p}<0,0001$ & 0,132 \\
\hline Bachillerato o más & 0,171 & 1,186 & {$[1,14,1,24]$} & $\mathrm{p}<0,0001$ & 0,058 \\
\hline \multicolumn{6}{|l|}{ En unión } \\
\hline $\mathrm{No}+$ & - & - & - & - & \\
\hline $\mathrm{Si}$ & $-0,006$ & 0,994 & {$[0,97,1,02]$} & 0,646 & $-0,003$ \\
\hline \multicolumn{6}{|l|}{ Realiza actividad económica } \\
\hline $\mathrm{No}+$ & - & - & - & - & \\
\hline $\mathrm{Si}$ & 0,133 & 1,142 & {$[1,10,1,18]$} & $\mathrm{p}<0,0001$ & 0,066 \\
\hline \multicolumn{6}{|l|}{ Realiza actividad económica* Sexo } \\
\hline Hombre que No tiene actividad económica+ & - & - & - & - & \\
\hline Mujer que realiza actividad económica & 0,206 & 1,229 & {$[1,14,1,32]$} & $\mathrm{p}<0,0001$ & 0,041 \\
\hline \multicolumn{6}{|l|}{ Año } \\
\hline $2012+$ & - & - & - & - & \\
\hline 2013 & 0,015 & 1,015 & {$[0,97,1,06]$} & 0,471 & 0,006 \\
\hline 2014 & 0,104 & 1,109 & {$[1,07,1,15]$} & $\mathrm{p}<0,0001$ & 0,042 \\
\hline 2015 & 0,088 & 1,092 & {$[1,05,1,14]$} & $\mathrm{p}<0,0001$ & 0,036 \\
\hline 2016 & 0,009 & 1,009 & {$[0,97,1,05]$} & 0,660 & 0,004 \\
\hline Constante & $-2,905$ & 0,055 & {$[0,05,0,06]$} & $\mathrm{p}<0,0001$ & - \\
\hline $\begin{array}{l}\text { centaje de casos correctamente clasificados } \\
\text { eba de bondad de ajuste Hosmer-Lemeshow }\end{array}$ & $\begin{array}{l}99,01 \% \\
6,810\end{array}$ & 0,078 & & & \\
\hline
\end{tabular}

\section{Discusión}

Los resultados obtenidos permiten confirmar que la mortalidad por suicidio es mayor para hombres( ${ }^{(8)}$. Esta diferencia entre el comportamiento suicida, es decir, la mayor representación femenina en comportamiento de suicidio no fatal y la mayor mortalidad masculina se ha descrito como la paradoja del género( ${ }^{(9)}$. Una posible explicación de esto es que en general, los hombres utilizan métodos más efectivos para quitarse la vida(10), que se refleja en que los métodos masculinos más utilizados fueron el ahorcamiento y el arma de fuego, para las mujeres fueron el ahorcamiento y el envenenamiento; este último es considerado un método menos letal(11,12).

La edad es otro factor importante del suicidio dado que acarrea una mayor carga de la enfermedad por el creciente número de años de vida perdidos por muerte prematura(5). Se observó que tener algún grado de escolaridad incrementó la posibilidad de fallecer por suicidio; la evidencia sobre dicha relación es todavía inconsistente, ya que en el estudio de Xavier, et al., se encontró una asociación 
inversa entre la escolaridad y el suicidio(13) y en otros, un bajo logro educativo se asoció con mayor riesgo de suicidio(14,15). Realizar actividad económica incrementó la posibilidad de fallecer por suicidio, especialmente en mujeres. Esta relación se ha estudiado previamente, pero sobre todo son el desempleo y el subempleo aquellos que tienen una relación más directa con el suicidio(16,17).

No obstante las contribuciones realizadas en esta investigación, se debe considerar algunas limitaciones del estudio. Se trabajó con las estadísticas vitales de mortalidad, las cuales no tienen información sobre la salud mental de las personas o sobre otros factores de riesgo del suicidio mencionados previamente(6). A pesar de que se ha reportado errores asociados con la calidad y cobertura de las estadísticas vitales de mortalidad en Latinoamérica y El Caribe(18), que pueden generar subregistro de este evento; en general se considera que en México la información permite realizar estimaciones confiables(19). En cuanto a la actividad económica, no fue posible conocer qué tipo de actividad realizaban las personas fallecidas. Otra limitante adicional, es que este tipo de información transversal no permite analizar los posibles factores causales del suicidio consumado, sólo su asociación con dichas variables.

Este estudio tiene la ventaja que utiliza una fuente de información, y un acercamiento al fenómeno poco explorado, pese a su riqueza en datos y temporalidad. Los resultados se enfocaron en los factores asociados con el suicidio consumado, y dado que es un fenómeno prevenible que tiene una alta carga de la enfermedad(5).

\section{Conclusión}

Se espera que este estudio aporte mayor evidencia para generar políticas de prevención y atención oportuna que disminuyan el impacto de este evento en la sociedad.

\section{Financiación}

El presente estudio fue autofinanciado.

\section{Conflicto de intereses}

Ninguno declarado por el autor.

\section{Referencias}

1. Organización Mundial de la Salud. Prevención del suicidio un imperativo global. Washington D.C.: OMS; 2014.

2. Organización Panamericana de la Salud. Mortalidad por suicidio en las Américas. Informe regional. Washington D.C.: OPS; 2014.

3. Borges G, Benjet C, Orozco R, Medina-Mora ME. The growth of suicide ideation, plan and attempt among young adults in the Mexico City metropolitan area. Epidemiology and Psychiatric Sciences. 2017;26(6):635-643.

4. Borges G, Rosovsky H, Gómez C, Gutiérrez R. Epidemiología del suicidio en México de 1970 a 1994. Salud pública Méx. 1996;38:197-206.

5. Institute for Health Metrics and Evaluation. GBD Compare Data Visualization. Washington: GBD; 2017.

6. Borges G, Orozco R, Villatoro J, Medina-Mora ME, Fleiz C, Díaz-Salazar J. Suicide ideation and behavior in Mexico: Encodat 2016. Salud pública Méx. 2019;61:6-15.

7. World Health Organization. International Statistical Classification of Diseases and Related Health Problems 10th Revision. Geneva: WHO; 2010.

8. Turecki G, Brent DA. Suicide and suicidal behavior. The Lancet. 2016;387:1227-39.

9. Freeman A, Mergl R, Kohls E, Székely A, Gusmao R, Arensman E, et al. A cross-national study on gender differences in suicide intent. BMC psychiatry. 2017;17(1):234.

10. Jung S, Lee D, Park S, Lee K, Kweon YS, Lee EJ, et al. Gender differences in Korean adolescents who died by suicide based on teacher reports. Child and adolescent psychiatry and mental health. 2019;13(1):12.

11. Jamison EC, Bol KA. Previous Suicide Attempt and Its Association With Method Used in a Suicide Death. American Journal of Preventive Medicine. 2016;51(5):S226-S233.

12. Shojaei A, Moradi S, Alaeddini F, et al. Association between suicide method, and gender, age, and education level in Iran over 2006-2010. Asia-Pacific Psychiatry. 2014;6:18-22.

13. Xavier N, Gomes C, Nazareth S, Alves L, Werner J. Suicide rates in the State of Rio Grande do Sul, Brazil: association with socioeconomic, cultural, and agricultural factors. Cad Saúde Pública. 2006;22(12):2611-21.

14. Borges G, Nock MK, Medina-Mora ME, Benjet C, Lara C, Chiu WT, et al. The epidemiology of suicide-related outcomes in Mexico. Suicide and Life-Threatening Behavior. 2007;37(6):627-40.

15. Huang $X$, Ribeiro JD, Musacchio KM, Franklin JC. Demographics as predictors of suicidal thoughts and behaviors: A meta-analysis. PlosOne. 2017;12(7):e0180793.

16. Borges G, Orozco R, Medina-Mora ME. Índice de riesgo para el intento suicida en México. Salud pública Méx. 2012;54:595-606.

17. Kyoung-Bok M, Shin-Goo P, Sang Hee H, Jin-Young M. Precarious employment and the risk of suicidal ideation and suicide attempts. Preventive Medicine. 2015;71:72-76.

18. Palloni A, Pinto-Aguirre G. Adult mortality in Latin America and the Caribbean. In: Rogers RG, Crimmins EM, editors. International handbook of adult mortality. Dordrecht: Springer Netherlands; 2011. p. 101-32.

19. Bhalla K, Harrison JE, Shahraz S, Fingerhut LA. Availability and quality of cause-of-death data for estimating the global burden of injuries. Bulletin of the World Health Organization. 2010;88(11):831-838. 